\title{
Japanese consensus guidelines for pediatric nuclear medicine
}

\author{
Part 1: Pediatric radiopharmaceutical administered doses (JSNM pediatric dosage \\ card). Part 2: Technical considerations for pediatric nuclear medicine imaging \\ procedures
}

The Japanese Society of Nuclear Medicine - Optimization Committee for Pediatric Nuclear Medicine Studies • Kiyoshi Koizumi - Hidekazu Masaki - Hiroshi Matsuda • Mayuki Uchiyama • Mitsuo Okuno - Eiji Oguma Hiroshi Onuma $\cdot$ Kimio Kanegawa $\cdot$ Shinichi Kanaya $\cdot$ Hiroshi Kamiyama Kensuke Karasawa Masayuki Kitamura - Tetsuo Kida $\cdot$ Tatsuo Kono $\cdot$ Chisato Kondo $\cdot$ Masayuki Sasaki $\cdot$ Hitoshi Terada $\cdot$ Atsushi Nakanishi - Teisuke Hashimoto - Hiroshi Hataya - Shin-ichiro Hamano - Keishi Hirono • Yukihiko Fujita $\cdot$ Ken Hoshino $\cdot$ Masayuki Yano $\cdot$ Seiichi Watanabe

Received: 11 February 2014 / Accepted: 13 February 2014/Published online: 20 March 2014

(c) The Japanese Society of Nuclear Medicine 2014

\begin{abstract}
The Japanese Society of Nuclear Medicine has recently published the consensus guidelines for pediatric nuclear medicine. This article is the English version of the guidelines. Part 1 proposes the dose optimization in pediatric nuclear medicine studies. Part 2 comprehensively discusses imaging techniques for the appropriate conduct of pediatric nuclear medicine procedures, considering the characteristics of imaging in children.
\end{abstract}

\footnotetext{
K. Koizumi (凹)

Department of Radiology, Hachioji Medical Center, Tokyo

Medical University, 1163 Tate-machi, Hachioji,

Tokyo 193-0998, Japan

e-mail: kkoi@tokyo-med.ac.jp

H. Masaki · M. Kitamura

National Center for Child Health and Development, Tokyo, Japan

H. Matsuda $\cdot$ M. Sasaki

National Center of Neurology and Psychiatry, Tokyo, Japan

M. Uchiyama

The Jikei University Hospital, Tokyo, Japan

M. Okuno

Nihon University Hospital, Tokyo, Japan

E. Oguma $\cdot$ S. Hamano

Saitama Children's Medical Center, Saitama, Japan

H. Onuma

Nihon University Itabashi Hospital, Tokyo, Japan

K. Kanegawa

Aichi Children's Health and Medical Center, Aichi, Japan
}

Overview of the guidelines

Objectives and background of the guidelines

Nuclear medicine studies are important examinations that produce images of organ function and the pathophysiology of diseases, information that cannot be obtained by other diagnostic imaging methods. Nuclear medicine studies,

\footnotetext{
S. Kanaya

Tokyo Women's Medical University Hospital, Tokyo, Japan

H. Kamiyama · K. Karasawa · Y. Fujita

Nihon University School of Medicine, Tokyo, Japan

T. Kida

Shiga University of Medical Science Hospital, Shiga, Japan

T. Kono $\cdot$ H. Hataya

Tokyo Metropolitan Children's Medical Center, Tokyo, Japan

C. Kondo

Tokyo Women's Medical University, Tokyo, Japan

H. Terada

Toho University Sakura Medical Center, Chiba, Japan

A. Nakanishi

Juntendo University Shizuoka Hospital, Shizuoka, Japan

T. Hashimoto

Dokkyo Medical University, Tochigi, Japan

K. Hirono

Yokohama City University Hospital, Kanagawa, Japan
} 
like other radiological examinations, cause medical radiation exposure. Therefore, "justification of practice" and "optimization of protection" should always be kept in mind, particularly in children because they are considered to be more sensitive to radiation than adults.

In Japan, pediatric doses were recommended by a subcommittee, the Medical and Pharmaceutical Committee, Japan Radioisotope Association in 1988 [1]. The European Association of Nuclear Medicine proposed the standardization of pediatric radiopharmaceutical administered doses in 1990 [2], and published new, more detailed guidelines for calculating doses entitled "Pediatric Dosage Card" in 2007 [3, 4]. The Society of Nuclear Medicine and Molecular Imaging also proposed appropriate radiopharmaceutical administered doses for pediatric nuclear medicine studies in 2010 as part of the project to reduce medical radiation exposure of children called "Image Gently" [5-7]. In 2011, an incident of intentional administration of excessive radioactivity to children in nuclear medicine studies at a hospital in Japan was disclosed. With this case as the springboard, the Japanese Society of Nuclear Medicine set up a committee to review the appropriate conduct of pediatric nuclear medicine studies, including the radiopharmaceutical administered doses, in 2012. After review for approximately 1 year, the committee has prepared consensus guidelines for the appropriate conduct of pediatric nuclear medicine studies.

Outline and structure of the guidelines

Generally, guidelines should be objective and neutral, based on high-level evidence. In pediatric nuclear medicine studies, however, it is difficult to accumulate a sufficient number of cases and thus prepare guidelines based on evidence-based medicine (EBM). Therefore, the present guidelines have been prepared based on consensus reached by experts who are thoroughly familiar with pediatric nuclear medicine studies.

The original guidelines are written in Japanese and consist of three parts [8]. Part 1 proposes the dose optimization in pediatric nuclear medicine studies. The method for calculating the doses is based on the above-mentioned European Association of Nuclear Medicine's guidelines. The computed doses are almost equivalent to those proposed by the Society of Nuclear Medicine and Molecular Imaging, and are generally lower than those conventionally used in Japan. There is a global trend to reduce radiation

\footnotetext{
K. Hoshino

Keio University Medical School, Tokyo, Japan

M. Yano

Shizuoka Children's Hospital, Shizuoka, Japan

S. Watanabe

Tsuchiura Kyodo General Hospital, Ibaraki, Japan
}

exposure in clinical practice, and awareness of dose reduction should be raised among health care workers involved in nuclear medicine studies. Maintenance and renewal of equipment as well as improvement of imaging techniques and image reading skills may be required when lower doses were administered. Part 2 comprehensively discusses imaging techniques for the appropriate conduct of pediatric nuclear medicine procedures, considering the characteristics of imaging in children. Part 3 provides pediatric illnesses for which nuclear medicine studies are highly useful and that may partly justify the use of radiopharmaceuticals causing radiation exposure in children. In this English version, Parts 1 and 2 have been translated.

Although the guidelines may have some shortcomings in content, they are based on the current consensus of the Committee. The guidelines are subject to revision in accordance with the global trend toward standardization of pediatric nuclear medicine studies.

\section{Part 1: Pediatric radiopharmaceutical administered doses}

Concepts and methods for calculation

In the report concerning the recommendation for pediatric doses published in 1988 in Japan [1], the characteristics of various methods for calculating doses are discussed and compared with each other. Most of the methods presented in the report are based on the adult doses, which were multiplied by certain coefficients related to age, weight, body surface area, etc., to derive the pediatric administered dose. The proposed formula recommended by the subcommittee is "Pediatric dose $(\mathrm{MBq})=$ adult dose $\times$ $($ age +1$) /($ age +7$)$ ". This formula has been widely used in the clinical practice of pediatric nuclear medicine studies in Japan since then. The adult dose included in the formula generally refers to the adult reference dose proposed by the Japanese Society of Nuclear Medicine [9]; however, it has not been uniform across the nation, since some medical institutions use other reference doses. In addition, weightbased calculation methods are considered more reasonable than age-based calculation methods, because the prevalence of overweight children has recently been increasing and sick children are likely to have a lower body weight than children of the same age with a normal body weight. Both formulas proposed by the European Association of Nuclear Medicine and the Society of Nuclear Medicine and Molecular Imaging, respectively, directly compute the administered dose from the body weight of the patient, not based on the adult administered dose. Especially the method proposed by the European Association of Nuclear Medicine is designed to yield weight-independent effective doses. 
Table 1 Class and baseline activity/minimum activity of radiopharmaceuticals

\begin{tabular}{|c|c|c|c|c|}
\hline \multirow[t]{2}{*}{ Nuclides } & \multirow[t]{2}{*}{ Radiopharmaceutical } & \multirow[t]{2}{*}{ Class } & \multicolumn{2}{|l|}{ Doses } \\
\hline & & & $\begin{array}{l}\text { Baseline } \\
\text { activity } \\
\text { (MBq) }\end{array}$ & $\begin{array}{l}\text { Minimum } \\
\text { activity } \\
\text { (MBq) }\end{array}$ \\
\hline \multirow[t]{6}{*}{$\mathrm{I}-123$} & $\mathrm{NaI}$ & $\mathrm{C}$ & 0.6 & 3 \\
\hline & IMP & $\mathrm{B}$ & 13.0 & 18 \\
\hline & MIBG (tumors) & B & 28.0 & 40 \\
\hline & MIBG (myocardium) & B & 7.9 & 16 \\
\hline & Iomazenil & B & 11.9 & 24 \\
\hline & BMIPP & B & 7.9 & 16 \\
\hline F-18 & FDG & B & 14.0 & 14 \\
\hline Ga-67 & Citrate & $\mathrm{B}$ & 5.6 & 10 \\
\hline \multirow[t]{20}{*}{ Tc-99m } & Albumin (cardiac pool) & B & 56.0 & 80 \\
\hline & $\begin{array}{l}\text { Tin colloid (liver and } \\
\text { spleen) }\end{array}$ & $\mathrm{B}$ & 5.6 & 15 \\
\hline & $\begin{array}{l}\text { Tin colloid (bone } \\
\text { marrow) }\end{array}$ & B & 21.0 & 20 \\
\hline & $\begin{array}{l}\text { Phytate (liver and } \\
\text { spleen) }\end{array}$ & B & 5.6 & 15 \\
\hline & MDP/HMDP & B & 35.0 & 40 \\
\hline & DMSA & A & 25.6 & 15 \\
\hline & DTPA & A & 34.0 & 20 \\
\hline & MAG3 & A & 34.0 & 20 \\
\hline & ECD & B & 32.0 & 110 \\
\hline & HMPAO & B & 51.8 & 100 \\
\hline & PMT & B & 10.5 & 20 \\
\hline & MAA & B & 13.2 & 10 \\
\hline & Pertechnetate (thyroid) & B & 5.6 & 10 \\
\hline & $\begin{array}{l}\text { Pertechnetate (gastric } \\
\text { mucosa) }\end{array}$ & $\mathrm{B}$ & 10.5 & 20 \\
\hline & $\mathrm{RBC}$ & B & 56.0 & 80 \\
\hline & $\begin{array}{l}\text { MIBI/tetrofosmin } \\
\text { (tumors) }\end{array}$ & B & 63.0 & 80 \\
\hline & $\begin{array}{l}\text { MIBI/tetrofosmin } \\
\text { (myocardial rest/stress } \\
\text { scan 2-day protocol } \\
\max ^{\mathrm{a}} \text { ) }\end{array}$ & B & 63.0 & 80 \\
\hline & $\begin{array}{l}\text { MIBI/tetrofosmin } \\
\text { (myocardial scan 1-day } \\
\left.\text { protocol first }^{\mathrm{b}}\right)\end{array}$ & B & 28.0 & 80 \\
\hline & $\begin{array}{l}\text { MIBI/tetrofosmin } \\
\text { (myocardial scan 1-day } \\
\left.\text { protocol second }^{\text {b }}\right)\end{array}$ & B & 84.0 & 160 \\
\hline & GSA & B & 13.2 & 26 \\
\hline Tl-201 & $\begin{array}{l}\text { Thallium chloride } \\
\text { (tumors) }\end{array}$ & B & 5.3 & 11 \\
\hline In-111 & Indium chloride & B & 5.3 & 11 \\
\hline
\end{tabular}

a An excessive dose may be calculated of a heavy patient compared to the conventionally administered dose; therefore, a smaller dose should be considered since this dose is the maximum

b Both the rest first and the stress first protocols are applicable. The second dose is two to three times the first dose
Table 2 Weight-dependent multiples for each class

\begin{tabular}{|c|c|c|c|}
\hline \multirow[t]{2}{*}{ Body weight (kg) } & \multicolumn{3}{|c|}{ Class } \\
\hline & A & B & $\mathrm{C}$ \\
\hline 3 & 1 & 1 & 1 \\
\hline 4 & 1.12 & 1.14 & 1.33 \\
\hline 6 & 1.47 & 1.71 & 2 \\
\hline 8 & 1.71 & 2.14 & 3 \\
\hline 10 & 1.94 & 2.71 & 3.67 \\
\hline 12 & 2.18 & 3.14 & 4.67 \\
\hline 14 & 2.35 & 3.57 & 5.67 \\
\hline 16 & 2.53 & 4 & 6.33 \\
\hline 18 & 2.71 & 4.43 & 7.33 \\
\hline 20 & 2.88 & 4.86 & 8.33 \\
\hline 22 & 3.06 & 5.29 & 9.33 \\
\hline 24 & 3.18 & 5.71 & 10 \\
\hline 26 & 3.35 & 6.14 & 11 \\
\hline 28 & 3.47 & 6.43 & 12 \\
\hline 30 & 3.65 & 6.86 & 13 \\
\hline 32 & 3.77 & 7.29 & 14 \\
\hline 34 & 3.88 & 7.72 & 15 \\
\hline 36 & 4 & 8 & 16 \\
\hline 38 & 4.18 & 8.43 & 17 \\
\hline 40 & 4.29 & 8.86 & 18 \\
\hline 42 & 4.41 & 9.14 & 19 \\
\hline 44 & 4.53 & 9.57 & 20 \\
\hline 46 & 4.65 & 10 & 21 \\
\hline 48 & 4.77 & 10.29 & 22 \\
\hline 50 & 4.88 & 10.71 & 23 \\
\hline $52-54$ & 5 & 11.29 & 24.67 \\
\hline $56-58$ & 5.24 & 12 & 26.67 \\
\hline $60-62$ & 5.47 & 12.71 & 28.67 \\
\hline $64-66$ & 5.65 & 13.43 & 31 \\
\hline 68 & 5.77 & 14 & 32.33 \\
\hline
\end{tabular}

After considering all the points mentioned above, we determined the calculation method, "JSNM pediatric dosage card", which is a modified version of the method proposed by the European Association of Nuclear Medicine (EANM pediatric dosage card), selecting radiopharmaceuticals that are routinely used for pediatric nuclear medicine studies in Japan, and considering the current dosage of the radiopharmaceuticals.

The pediatric administered dose is computed as follows using the class, baseline activity, and weight-dependent multiple as presented in Tables 1 and 2.

\section{Formula for calculation}

Administered activity $(\mathrm{MBq})=$ baseline activity in Table $1 \times$ weight-dependent multiple in Table 2 . 
If the calculated activity is smaller than the minimum recommended activity, the minimum activity should be administered.

If the calculated activity exceeds the adult dose specified at each institution, the adult dose should be administered.

\section{Examples of calculation}

(Ex. 1) Administration of Tc-99m-MAG3 to a patient weighing $6 \mathrm{~kg}$ :

$34.0 \times 1.47=50.0>20 \rightarrow$ The activity to be administered is $50.0 \mathrm{MBq}$

(Ex. 2) Administration of Tc-99m-ECD to a patient weighing $10 \mathrm{~kg}$ :

$32.0 \times 2.71=86.7<110 \rightarrow$ The activity to be administered is $110 \mathrm{MBq}$

(Ex. 3) Administration of Tc-99m-tetrofosmin (myocardial scan 2-day protocol) to a patient weighing $44 \mathrm{~kg}$ :

$63.0 \times 9.57=603>592$ (adult administered activity at the institution) $\rightarrow$ The activity to be administered is $592 \mathrm{MBq}$.

\section{Classifications of radiopharmaceuticals and definitions of factors in the formula}

Class: Classification for standardization of the effective doses

Class A Radiopharmaceuticals for renal studies Class C Sodium iodine (I-123) for thyroid studies Class B All other radiopharmaceuticals.

Baseline activity: Mostly equals the activity adopted by the Pediatric Task Group, the European Association of Nuclear Medicine, which was set based on the European adult administered activity. For some radiopharmaceuticals, the activity was originally determined based on the adult administered activity in Japan. These values are for calculation purposes and equal the activity to be administered to a child weighing $3 \mathrm{~kg}$.

Minimum activity: Mostly equals the minimum activity that was empirically determined by the European Association of Nuclear Medicine. For some radiopharmaceuticals, the activity was originally determined based on the pediatric administered activity in Japan.

Weight-dependent multiple: Factors dependent on body weight that were determined to obtain weight-independent effective doses

\section{Remarks}

The radiopharmaceuticals listed in Table 1 are administered intravenously, except for sodium iodine (I-123) which is administered orally. The activities to be administered in particular studies for which the radiopharmaceuticals are administered by other routes are individually presented in Part 3.

The guidelines generally cover children aged 15 years and younger. Not all of those aged 16 years and older, however, can be given the adult administered activities. The guidelines may have to be applied even to a child aged 16 years or older if he/she is considered a growing youth, and careful determination of the appropriate dosage is required.

\section{Part 2: Technical considerations for pediatric nuclear medicine imaging procedures}

General concepts

The pediatric radiopharmaceutical administered doses proposed in Part 1 are mostly lower than those that have been widely used in Japan. Attempts should be made to achieve good imaging performance with these lower doses [10].

Although it is theoretically possible to obtain some information, even with low doses of radiopharmaceuticals, by spending a longer time, too lengthy restraining of the body may cause unexpected body motions, resulting in completely unusable data. It is therefore important to keep children at rest during the examination and to prioritize the information to be collected within the limited time of the examination. If the quality of the images is judged to be insufficient due to body motions, imaging has to be repeated, but continuation of the examination may be impossible due to the mental status of the patient. To prevent such a situation, it is important to communicate with the patients before the examination and to monitor them carefully and talk to them frequently during the examination [11].

Prior to an examination, close communications with the concerned departments should be secured and the patient's background factors such as age, height, body weight, disease and symptoms should be fully grasped. It is preferable to prioritize the images to be taken and select the imaging method in advance, based on these background factors, and to use an individual protocol that is appropriate for the circumstances.

Special considerations

\section{Procedures during imaging}

During imaging, efforts should be made to keep the children at rest and to acquire appropriate images within a 
limited time. For this purpose, skilled manipulation, much experience, and special considerations and patience for children are required. Measures that are appropriate for the individual circumstances of each patient should be taken. Modifications of the routine imaging protocol or the preparation of imaging protocol variations may also be required.

Once a radiopharmaceutical is administered, imaging should not easily be discontinued in the course of the procedure, for example, because the patient is crying or has woken up. Utmost efforts should be made to complete the examination by trying to calm the patient down by appropriate means or allowing differences in the imaging time.

To be prepared for a sudden change in the medical condition of patients during the examination, rescue medications indicated for children, resuscitation equipment, oxygen, etc., as well as the proper medical environment and staff to deal with an unexpected accident, must be available.

\section{Infusion of radiopharmaceuticals}

Vascular access should be secured well in advance to make the best use of the limited examination time. If it is planned to administer a sedative, vascular access should be secured before the administration. After administration of the radiopharmaceutical through the intravenous line, a small amount may remain in the three-way stopcock or the syringe that was used, and the residual amount should be washed or flushed with an adequate volume of saline. Careful attention should be paid to the infusion of smallvolume radiopharmaceuticals and Tc-99m-MAA, which adheres to the walls of the tubes or syringe. For the measurement of the administered activity, a properly calibrated dose calibrator should be used.

\section{Restraining body motions}

Keeping children at rest during the examination is critical and ways must therefore be devised to restrain body motions. Some children fall asleep in a quiet examination room with dim lights. For immobilization, soft (for infants) or hard (for schoolchildren) Velcro fasteners, or elastic bandages of different widths may be used to maintain the positioning of the patients during lengthy examinations, without applying excessive pressure. The clearances between adjoining sites of the body should be secured by placing towels between the arm or leg and the trunk, to facilitate the correct interpretation of the images. Sometimes, immobilization is counterproductive. In children who can understand to some extent, allowing them to watch their favorite video program or to hold a favorite toy or stuffed animal may help to distract and relax them.

\section{Sedation and anesthesia}

If immobilization using the above-mentioned tips is not sufficient to restrain body motions, sedation or anesthesia may be necessary. Since sedation and anesthesia are somewhat invasive, their justification and optimization should be considered, as with the use of radiopharmaceuticals. The staff involved in imaging examinations must monitor the child for respiratory depression or misswallowing from the time of sedation or anesthesia until the end of the examination, and ask the concerned staff to continue careful observation of the child after the examination is over. Participation of an anesthesiologist in the imaging team may be considered [12].

\section{Equipment and analysis}

Optimal imaging of children requires special considerations for the appropriate choices of equipment, image acquisition and analytical methodologies. The dose of the radiopharmaceutical conventionally used at the institution should be compared with the dose proposed by the guidelines and the acquisition conditions may be re-determined. Especially for examinations with limitations of the acquisition time and timing, such as dynamic imaging and SPECT, prior evaluation of the acquisition protocols is important.

In addition to changes in the acquisition time and pixel size, the choice of an appropriate collimator is critical. To detect detailed abnormalities, a high-resolution collimator may be the most appropriate, even if a slightly longer imaging time is required. To acquire a less noisy image during a limited time, on the other hand, a high-sensitivity collimator may be a good choice. An appropriate collimator is not only selected based on the visual quality of the image, but also on the ability to detect abnormal findings or the feasibility of diagnosis.

For SPECT imaging of a small child, the detectors cannot get close to the patient on an imaging bed designed for adults. The use of a narrow bed that is specially designed for children may be preferable. To examine small organs, the acquisition of magnified images can help with image interpretation. The magnification of an image, however, reduces the information density, resulting in deterioration of the image quality and thereby requiring a longer acquisition time.

If body motions of patients cannot be properly restrained, the use of motion correction programs or the need for reacquisition may be considered after the linogram/sinogram has been reviewed. However, motion correction programs should not be overestimated and if large or frequent body motions are observed, reacquisition should be made. Processing of inappropriate data including 
body motions, which may lead to a misdiagnosis, should be avoided.

To handle lower radiation exposure to children and shorter imaging time, the use of an advanced reconstruction method should also be considered. Filtering of images, 3DOSEM (ordered-subset expectation maximization), which is known to reduce statistical noise, and corrections of scatter, attenuation and resolution are helpful to improve image quality.

The equipment to be used has to be checked periodically and they should be adequately maintained and managed. If natural aging degradation of the imaging system such as its sensitivity and uniformity is noted and appropriate imaging is considered to be impossible, prompt renewal of the equipment should be considered. The administered doses of radiopharmaceuticals should not be increased to compensate for the deterioration of equipment.

\section{Handling of urination}

Imaging examinations of children should start after a diaper change or urination. Incomplete urination may cause body motions due to the urge to urinate and cause obscuration of the targeted site due to a full bladder. If spontaneous urination is incomplete or urinary incontinence during imaging is expected, a balloon catheter may be introduced beforehand. The catheter should be covered with a lead plate during imaging to avoid including unnecessary signals in the image due to the urinary catheter.

Even if a balloon catheter is placed, not all urine may be discharged through the catheter, and some urine may leak between the urethra and the catheter, causing contamination of diapers and the body surface with urine. Although many schoolchildren are able to spontaneously discharge urine, and urine in the bladder is less likely to affect the image quality, some children may feel the need to urinate sooner due to excessive nervousness, resulting in discontinuation of the examination.

Most radiopharmaceuticals are excreted into the urine. Therefore, the bladder wall is the most exposed to radiation in general. Voiding prompted by adequate hydration and continuous urinary drainage with a balloon catheter are preferable to reduce radiation exposure.

Frequent diaper changing may be helpful to reduce radiation exposure to the gonads [13].

\section{SPECT/CT and PET/CT}

Although additional CT images may improve the diagnostic performance of SPECT or PET alone, the use of
SPECT/CT or PET/CT should be determined after considering the increase in radiation exposure from CT scans. The unnecessary creation of high-resolution CT images should then be avoided, and radiation exposure from CT scans should be minimized by effectively using exposure reduction techniques.

\section{References}

1. Subcommittee for Standardization of Radionuclide Imaging, Medical and Pharmaceutical Committee, Japan Radioisotope Association. Recommendation for pediatric dose in nuclear imaging (in Japanese). Radioisotopes. 1988;37:627-32.

2. Paediatric Task Group European Association Nuclear Medicine Members, Piepsz A, Hahn K, Roca I, Clofetta G, Toth G, Gordon $\mathrm{I}$, et al. A radiopharmaceuticals schedule for imaging in paediatrics. Eur J Nucl Med. 1990;17:127-9.

3. Jacobs H, Thierens H, Piepsz A, Bacher K, Van de Wiele C, Ham $\mathrm{H}$, et al. Optimised tracer-dependent dosage cards to obtain weight-independent effective doses. Eur J Nucl Med Mol Imaging. 2005;32:581-8.

4. Lassmann M, Biassoni L, Monsieurs M, Franzius C, Jacobs F. The new EANM paediatric dosage card. Eur J Nucl Med Mol Imaging. 2007;34:796-8.

5. Gelfand MJ, Parisi MT, Treves ST. Pediatric radiopharmaceutical administered doses: 2010 North American consensus guidelines. J Nucl Med. 2011;52:318-22.

6. Fahey FH, Treves ST, Adelstein SJ. Minimizing and communicating radiation risk in pediatric nuclear medicine. J Nucl Med. 2011;52:1240-51.

7. Zanzonico P. Virtual reality for dose optimization in pediatric nuclear medicine. J Nucl Med. 2011;52:1845-7.

8. Optimization Committee for Pediatric Nuclear Medicine Studies, the Japanese Society of Nuclear Medicine: The Japanese consensus guidelines for pediatric nuclear medicine. http://www. jsnm.org/files/pdf/guideline/2013/PediatricNuclMedGuideline12-3.pdf (in Japanese). Accessed 6 Jan 2014.

9. Review Committee on Appropriate Use of Radiopharmaceuticals, the Japanese Society of Nuclear Medicine: The Guidelines for Appropriate Use of Radiopharmaceuticals, Sponsored by the Ministry of Health, Labour and Welfare in Fiscal Years 2001 and 2002. http://www.jsnm.org/files/pdf/guideline/iyakuhin_gaidor ain.pdf (in Japanese). Accessed 6 Jan 2014.

10. Ishii K. Practical pediatric nuclear medicine (in Japanese). In: Hisada K, Tonami N, Kubo A, editors. A new textbook of clinical nuclear medicine. 3rd ed. Tokyo: Kanehara \& Co., Ltd.; 1999. p. 661-76.

11. Treves ST. Introduction. In: Treves ST, editor. Pediatric nuclear medicine/PET. 3rd ed. New York: Springer; 2007. p. 1-15.

12. Treves ST, Baker A, Fahey FH, Cao X, Davis RT, Drubach LA, et al. Nuclear medicine in the first year of life. J Nucl Med. 2011;52:905-25.

13. Yano M. Visual pediatric nuclear Medicine. Tokyo: Medical Review Co., Ltd.; 2005. 